\title{
Antibacterial Activity of Rhus javanica against Methicillin-Resistant Staphylococcus aureus
}

\author{
Yong-Ouk You, ${ }^{1,2}$ Na-Young Choi, ${ }^{3}$ Sun-Young Kang, ${ }^{1}$ and Kang-Ju Kim ${ }^{2,4}$ \\ ${ }^{1}$ Department of Oral Biochemistry, School of Dentistry, Wonkwang University, Iksan 570-749, Republic of Korea \\ ${ }^{2}$ Wonkwang Research Institute for Food Industry, Iksan 570-749, Republic of Korea \\ ${ }^{3}$ College of Education, Wonkwang University, Iksan 570-749, Republic of Korea \\ ${ }^{4}$ Department of Oral Microbiology, School of Dentistry, Wonkwang University, Iksan 570-749, Republic of Korea
}

Correspondence should be addressed to Kang-Ju Kim; kaob@wku.ac.kr

Received 22 July 2013; Revised 28 August 2013; Accepted 28 August 2013

Academic Editor: Tong Ho Kang

Copyright (C) 2013 Yong-Ouk You et al. This is an open access article distributed under the Creative Commons Attribution License, which permits unrestricted use, distribution, and reproduction in any medium, provided the original work is properly cited.

\begin{abstract}
In the present study, the leaves of Rhus javanica ( $R$. javanica) were extracted with ethanol, and we investigated the antimicrobial activity of the ethanol extract of R. javanica against methicillin-resistant Staphylococcus aureus (MRSA). Control groups were treated with media containing $0.1 \%$ DMSO. The ethanol extract of $R$. javanica inhibited the growth of MRSA at concentrations ranging from 0.05 to $0.2 \mathrm{mg} / \mathrm{mL}$ and inhibited acid production at concentrations higher than $0.1 \mathrm{mg} / \mathrm{mL}(P<0.05)$. MRSA biofilm formation was determined by scanning electron microscopy and safranin staining. The ethanol extract of $R$. javanica inhibited the formation of MRSA biofilms at concentrations higher than $0.05 \mathrm{mg} / \mathrm{mL}$. In confocal laser scanning microscopy, high concentration $(0.4-$ $1.6 \mathrm{mg} / \mathrm{mL}$ ) of $R$. javanica extract showed bactericidal effect in a dose-dependent manner. In real-time PCR analysis, $R$. javanica extract showed the inhibition of the genetic expression of virulence factors such as mecA, sea, agr $A$, and $\operatorname{sar} A$ in MRSA. Preliminary phytochemical analysis revealed the strong presence of phenolics. These results suggest that $R$. javanica may be a useful medicinal plant for inhibiting MRSA, which may be related to the presence of phenolics in the $R$. javanica extract.
\end{abstract}

\section{Introduction}

Staphylococcus aureus has been reported to cause many diseases, from mild skin infections to more serious invasive infections such as suppuration, abscess formation, pneumonia, and even fatal septicemia in human beings [1]. Prior to the antibiotic era, S. aureus was associated with $80 \%$ mortality, but the advent of the earliest antimicrobial substances, such as penicillin, contributed to a reduction in mortality [2]. It was reported that over $94 \%$ of $S$. aureus strains were susceptible to penicillin at that time. However, antibiotic resistance in $S$. aureus has rapidly developed due to the widespread use of antibiotics [2, 3]. Methicillin-resistant Staphylococcus aureus (MRSA) was isolated in the early 1960s [4]. MRSA is resistant to not only methicillin and other $\beta$-lactams but also many other antibacterial agents such as macrolides and aminoglycosides [5,6]. MRSA is one of important causes of modern chronic infectious diseases $[3,5,6]$. One such antibioticresistant mechanism involves the production of $\beta$-lactamase or penicillin-binding protein 2a (PBP 2a) by MRSA [2]. MRSA forms biofilms on implanted medical devices such as catheters, plates, screws, artificial joints, and cardiac valves in patients $[7,8]$. Biofilm formation by MRSA has been reported to increase the antibiotic resistance of MRSA [9]. MRSA metabolizes carbohydrates to produce organic acids, which can stimulate biofilm formation [10,11]. At present, MRSA is emerging worldwide as one of the most important hospital and community pathogens due to its multidrug resistance. Therefore, new strategies are required to deal with MRSAassociated infections. Recently, scientists have focused on certain natural products with antipathogenic potential as candidates for new antibiotic substances $[12,13]$.

Rhus javanica ( $R$. javanica) is a member of Anacardiaceae and is mainly produced in Korea, China, and Japan $[14,15]$. It has been traditionally used to treat dysentery and diarrhea [15]. However, there is little scientific evidence regarding the effect of $R$. javanica on MRSA. In the course of screening for the antibacterial activities of natural products against 
MRSA, we recently found that extracts of $R$. javanica exhibit antibacterial activity against this pathogen. In the present study, we show that $R$. javanica has antimicrobial activity against and inhibits biofilm formation by MRSA. We also show the presence of major phytochemicals in R. javanica.

\section{Materials and Methods}

2.1. Materials. Brain heart infusion (BHI) broth was purchased from Difco Laboratories (Detroit, MI, USA). Glucose and dimethyl sulfoxide (DMSO) were obtained from Sigma Co. (St. Louis, MO, USA). MRSA ATCC 33591 was purchased from the American Type Culture Collection (ATCC; Manassas, VA, USA).

2.2. Plant Material and Extraction. The leaves of R. javanica were obtained from the oriental drug store Dae Hak Yak Kuk (Iksan, South Korea). The identity of the specimen was confirmed by Dr. Bong-Seop Kil at the Department of Natural Science, Wonkwang University (Iksan, South Korea). A voucher specimen (number 05-11) has been deposited at the Herbarium of the Department of Oral Biochemistry in Wonkwang University. Dried leaves $(600 \mathrm{~g})$ of $R$. javanica were soaked in $3000 \mathrm{~mL}$ of $90 \%$ ethanol for $72 \mathrm{~h}$ at room temperature. The extracted solution was filtered and evaporated under reduced pressure to yield an ethanol extract of $42.4 \mathrm{~g}$ (7.1\%). After the extract was thoroughly dried to facilitate complete removal of the solvent, the dry extract was dissolved in DMSO to give the desired stock solution. The final concentration of DMSO applied to culture systems was adjusted to $0.1 \%(\mathrm{v} / \mathrm{v})$, which did not interfere with the testing system. Control groups were treated with media containing $0.1 \%$ DMSO.

2.3. Bacterial Growth and Acid Production. Bacterial growth was determined using a modification of a previously described method $[16,17]$. The growth of MRSA was examined at $37^{\circ} \mathrm{C}$ in $0.95 \mathrm{~mL}$ of $\mathrm{BHI}$ broth containing various concentrations of the ethanol extract of $R$. javanica. These tubes were inoculated with $0.05 \mathrm{~mL}$ of an overnight culture grown in BHI broth (final: $5 \times 10^{5}$ colony-forming units $(\mathrm{CFU}) / \mathrm{mL}$ ), and incubated at $37^{\circ} \mathrm{C}$. After $24 \mathrm{~h}$ of incubation, the optical density (OD) of cells was measured spectrophotometrically at $550 \mathrm{~nm}$, and the $\mathrm{pH}$ of the cultures was determined using a pH meter (Corning Inc., Corning, NY, USA). Three replicates were measured for each concentration of the test extract. NaF (1\%) was used as a positive control.

2.4. Biofilm Assay. The biofilm assay was based on a method described previously $[18,19]$. $R$. javanica extract was added to $\mathrm{BHI}$ broth containing $1 \%$ glucose in $35 \mathrm{~mm}$ polystyrene dishes (Nunc, Copenhagen, Denmark). The cultures were then inoculated with a seed culture of MRSA (final: $5 \times$ $10^{5} \mathrm{CFU} / \mathrm{mL}$ ). After cultivating for $48 \mathrm{~h}$ at $37^{\circ} \mathrm{C}$, the supernatant was removed completely, and the dishes were rinsed with distilled $\mathrm{H}_{2} \mathrm{O}$. The biofilm formed on the surface of the dishes was also stained with $0.1 \%$ safranin, and photographed.
2.5. Scanning Electron Microscopy (SEM). The biofilm on 35$\mathrm{mm}$ polystyrene dishes was also determined by SEM using a modification of a previously described method [20]. The biofilm formed on the dishes was rinsed with distilled $\mathrm{H}_{2} \mathrm{O}$ and fixed with $2.5 \%$ glutaraldehyde in $0.1 \mathrm{M}$ sodium cacodylate buffer $(\mathrm{pH} 7.2)$ at $4^{\circ} \mathrm{C}$ for $24 \mathrm{~h}$. After gradual dehydration with ethyl alcohol $(60 \%, 70 \%, 80 \%, 90 \%, 95 \%$, and $100 \%)$, the sample was freeze-dried. The specimens were then sputtercoated with gold (108A sputter coater, Cressington Scientific Instruments Inc., Watford, England, UK). For observation, a JSM-6360 SEM (JEOL, Tokyo, Japan) was used.

2.6. Bactericidal Effect of $R$. javanica Extract. Bactericidal effect of $R$. javanica extract was determined by confocal laser scanning microscopy. The cultured MRSA in BHI was diluted using BHI media to approximately $1 \times 10^{7} \mathrm{CFU} / \mathrm{mL}$. The bacteria $\left(1 \times 10^{7} \mathrm{CFU} / \mathrm{mL}\right)$ were treated with high concentrations $(0.2-1.6 \mathrm{mg} / \mathrm{mL})$ of $R$. javanica extract at $37^{\circ} \mathrm{C}$ under aerobic conditions. After $30 \mathrm{~min}$ of incubation, the bacteria were washed with PBS and stained with LIVE/DEAD BacLight Bacterial Viability Kit (Molecular Probes, Eugene, OR, USA), prepared according to the manufacturer's instructions, for $15 \mathrm{~min}$. Stained bacteria were observed confocal laser scanning microscopy (LSM 510, Zeiss, Germany). This method is based on two nucleic acid stains: green fluorescent SYTO 9 stain and red fluorescent propidium iodide stain which differ in their ability to penetrate healthy bacterial cells. SYTO 9 stain labels live bacteria, in contrast propidium iodide penetrates only bacteria with damaged membranes.

2.7. Real-Time Polymerase Chain Reaction (PCR) Analysis. To determine the effect of $R$. javanica extract on gene expression, a real-time PCR assay was performed. The subminimal inhibitory concentration $(0.01-0.1 \mathrm{mg} / \mathrm{mL})$ of $R$. javanica extract was used to treat and culture MRSA for $24 \mathrm{~h}$. Total RNA was isolated from S. mutans by using Trizol reagent (Gibco-BRL) according to the manufacturer's instructions. Then, cDNA was synthesized using a reverse transcriptase reaction (Superscript; Gibco-BRL). The DNA amplifications were carried out using an ABI-Prism 7,000 Sequence Detection System with Absolute QPCR SYBR Green Mixes (Applied Bio systems Inc., Foster City, CA, USA). The primer pairs that were used in this study were described by previous reports [21-23] and are listed in Table 1. $16 \mathrm{~S}$ rRNA was used as an internal control.

2.8. Phytochemical Screening. Phytochemical tests of the extract were performed as previously described [24, 25]. Mayer's reagent was used for alkaloids, ferric chloride reagent for phenolics, Molish test for glycosides, Biuret reagent for peptides, $\mathrm{Mg}-\mathrm{HCl}$ reagent for flavonoids, Libermann-Burchard reagent for steroids, and silver nitrate reagent for organic acids.

2.9. Statistical Analysis. All experiments were performed in triplicate. Data were analyzed using the Statistical Package for Social Sciences (SPSS, Chicago, IL, USA). The data are expressed as the mean \pm standard deviation values. The 
TABle 1: Nucleotide sequences of primer used for real-time PCR in this study.

\begin{tabular}{|c|c|c|c|}
\hline Gene & Gene description & & Primer sequences $\left(5^{\prime}-3^{\prime}\right)$ \\
\hline \multirow{2}{*}{ 16S rRNA } & \multirow{2}{*}{ Normalizing internal standard } & Forward & ACTGGGATAACTTCGGGAAA \\
\hline & & Reverse & CGTTGCCTTGGTAAGCC \\
\hline \multirow{2}{*}{ mecA } & \multirow{2}{*}{ Penicillin binding protein $2^{\prime}$} & Forward & GTTAGATTGGGATCATAGCGTCATT \\
\hline & & Reverse & TGCCTAATCTCATATGTGTTCCTGTAT \\
\hline \multirow{2}{*}{ sea } & \multirow{2}{*}{ Staphylococcal enterotoxin A } & Forward & ATGGTGCTTATTATGGTTATC \\
\hline & & Reverse & CGTTTCCAAAGGTACTGTATT \\
\hline \multirow{2}{*}{$\operatorname{agr} A$} & \multirow{2}{*}{ Accessory gene regulator A } & Forward & TGATAATCCTTATGAGGTGCTT \\
\hline & & Reverse & CACTGTGACTCGTAACGAAAA \\
\hline \multirow{2}{*}{$\operatorname{sar} A$} & \multirow{2}{*}{ Staphylococcal accessary regulator A } & Forward & TGTTATCAATGGTCACTTATGCTG \\
\hline & & Reverse & TCTTTGTTTTCGCTGATGTATGTC \\
\hline
\end{tabular}

differences between the means of the experimental and control groups were evaluated by Student's $t$-test. Values of $P<0.05$ were considered statistically significant.

\section{Results}

3.1. Bacterial Growth Inhibition by R. javanica. In the present study, we investigated the antibacterial activity of the extract of $R$. javanica against MRSA. The bacteria were exposed to $0.01,0.05,0.1$, and $0.2 \mathrm{mg} / \mathrm{mL}$ of the ethanol extract of $R$. javanica. As seen in Figure 1, the extract (0.05$0.2 \mathrm{mg} / \mathrm{mL}$ ) showed antibacterial activity against MRSA in a dose-dependent manner and resulted in high MRSA growth inhibition at concentration $>0.1 \mathrm{mg} / \mathrm{mL}$ compared to the control group $(P<0.05)$. The positive control $(0.1 \% \mathrm{NaF})$ also indicated antibacterial activity. The minimum inhibitory concentration (MIC) for the ethanol extract of $R$. javanica is $0.1 \mathrm{mg} / \mathrm{mL}$. The determination of the MIC revealed the antimicrobial activity of the ethanol extract of $R$. javanica against MRSA.

3.2. Inhibitory Effect of $R$. javanica on Acid Production. Acid productions of the bacteria treated with $R$. javanica extract were also monitored. As summarized in Table 2, there was an obvious decrease in $\mathrm{pH}$ in the control group, but the decrease was substantially inhibited in the presence of the extract $(0.1-0.2 \mathrm{mg} / \mathrm{mL})$. The decrease in $\mathrm{pH}$ was also inhibited in the positive control group $(0.1 \%$ of $\mathrm{NaF})$. These results demonstrate that $R$. javanica extract may inhibit organic acid production in MRSA.

3.3. Inhibitory Effect of R. javanica on Biofilm Formation. We examined the inhibitory effect of $R$. javanica extract on MRSA biofilm formation by safranin staining. As shown in Figure 2, the extract of $R$. javanica $(0.05-0.2 \mathrm{mg} / \mathrm{mL})$ inhibited biofilm formation by MRSA. Biofilm formation was also inhibited in the presence of the positive control $(0.1 \% \mathrm{NaF})$. SEM photographs underline the results obtained by safranin staining (Figure 3). MRSA attached to and aggregated on the surface of polystyrene $35 \mathrm{~mm}$ dishes and visibly formed the biofilm in the control group, but biofilm formation was lower in the presence of $R$. javanica extract at concentrations higher

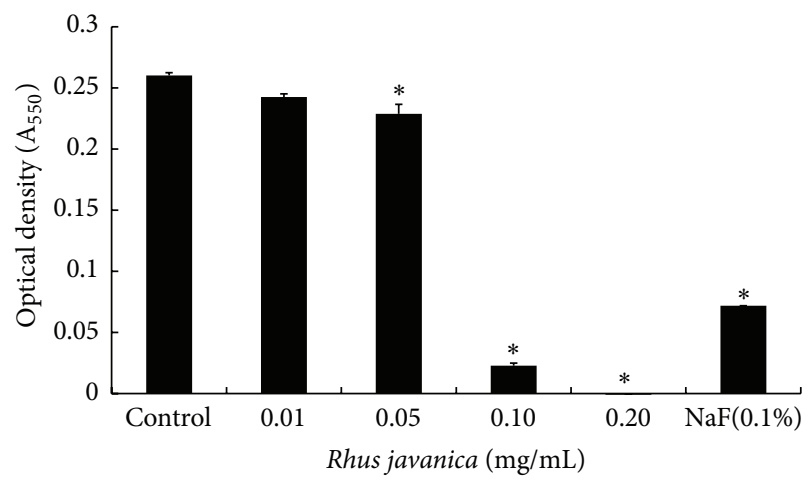

FIGURE 1: Effect of ethanol extract of R. javanica on the growth of MRSA. MRSA was inoculated into BHI broth with various concentrations of $R$. javanica and incubated for $24 \mathrm{~h}$ at $37^{\circ} \mathrm{C}$. The optical density $\left(A_{550}\right)$ was read using a spectrophotometer. Data are mean \pm standard deviation. ${ }^{*} P<0.05$ compared to the control group.

TABLE 2: Effect of ethanol extract of $R$. javanica on acid production by MRSA.

\begin{tabular}{lcc}
\hline $\begin{array}{l}\text { Concentration } \\
(\mathrm{mg} / \mathrm{mL})\end{array}$ & $\mathrm{pH}$ (before incubation) & $\mathrm{pH}$ (after incubation) \\
\hline Control & $7.20 \pm 0.05$ & $5.80 \pm 0.05^{1}$ \\
0.01 & $7.13 \pm 0.05$ & $5.13 \pm 0.20$ \\
0.05 & $7.13 \pm 0.04$ & $5.73 \pm 0.36^{*}$ \\
0.10 & $7.13 \pm 0.05$ & $6.67 \pm 0.23^{*}$ \\
0.20 & $7.13 \pm 0.00$ & $7.17 \pm 0.40^{*}$ \\
$\mathrm{NaF}(0.1 \%)$ & $7.20 \pm 0.00$ & $7.18 \pm 0.20^{*}$ \\
\hline${ }^{1} \mathrm{Data}(\mathrm{pH})$ are represented as mean \pm standard deviation. ${ }^{*} P<0.05$ \\
compared to the control group after incubation.
\end{tabular}

than $0.05 \mathrm{mg} / \mathrm{mL}$. Biofilm formation was also lower in the presence of the positive control.

3.4. Bacteriocidal Effect of $R$. javanica. Bactericidal effect of $R$. javanica extract was tested by confocal laser scanning microscopy (Figure 4 ). Bacterial viability was gradually decreased at high concentration $(0.4-1.6 \mathrm{mg} / \mathrm{mL})$ of $R$. javanica extract in a dose-dependent manner. 


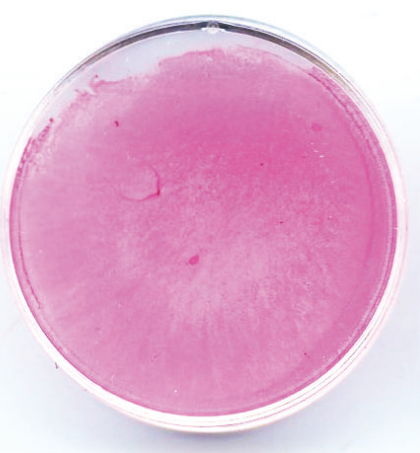

(a)

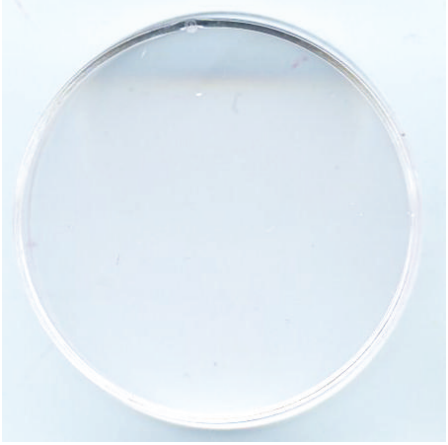

(d)

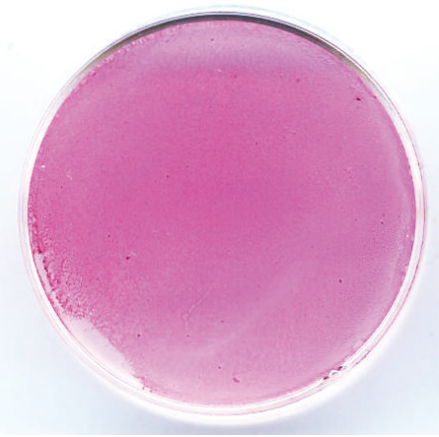

(b)

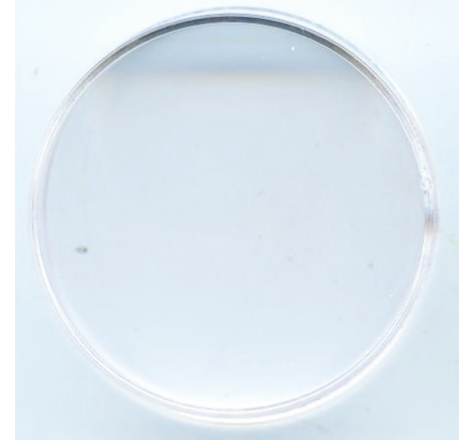

(e)

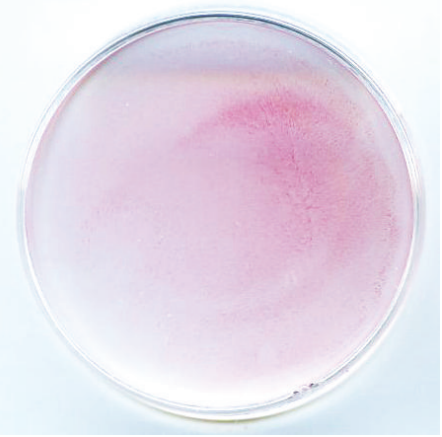

(c)

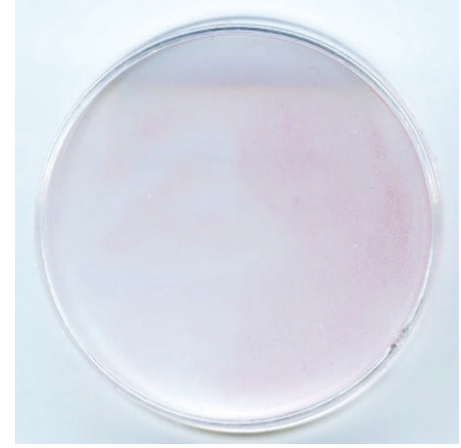

(f)

FIGURE 2: Effect of ethanol extract of R. javanica on biofilm formation by MRSA. MRSA was inoculated into BHI broth with various concentrations of $R$. javanica and incubated for $48 \mathrm{~h}$ at $37^{\circ} \mathrm{C}$. The biofilm formed on the surface of the dishes was also stained with $0.1 \%$ safranin and photographed. (a) Control; (b) $0.01 \mathrm{mg} / \mathrm{mL}$; (c) $0.05 \mathrm{mg} / \mathrm{mL}$; (d) $0.1 \mathrm{mg} / \mathrm{mL}$; (e) $0.2 \mathrm{mg} / \mathrm{mL}$; (f) positive control (0.1\% NaF).

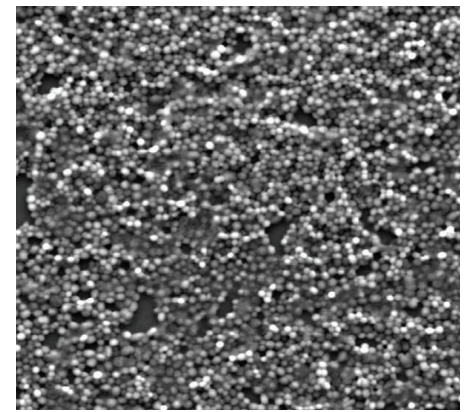

(a)

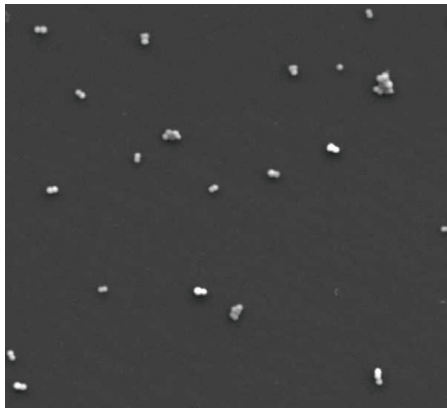

(d)

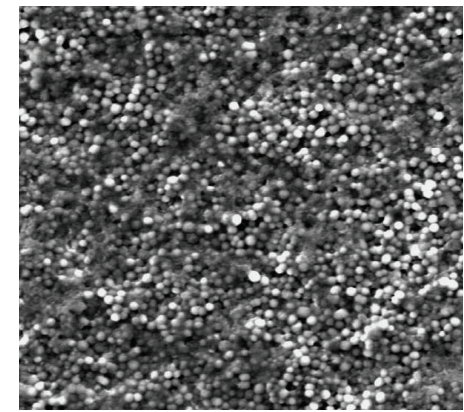

(b)

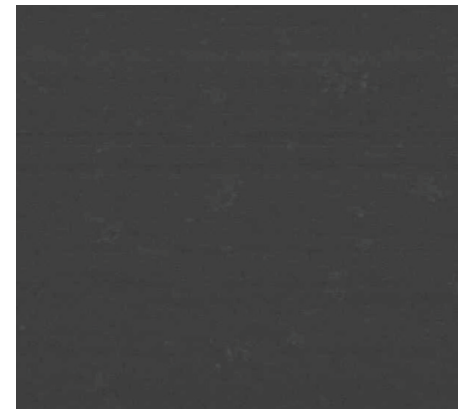

(e)

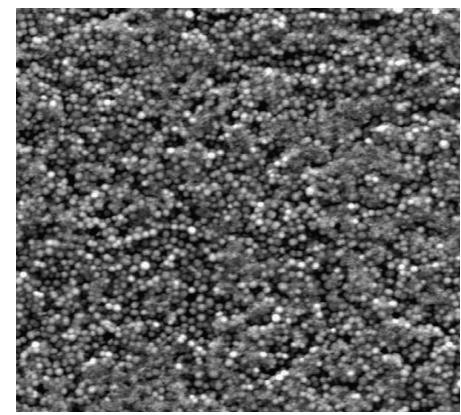

(c)

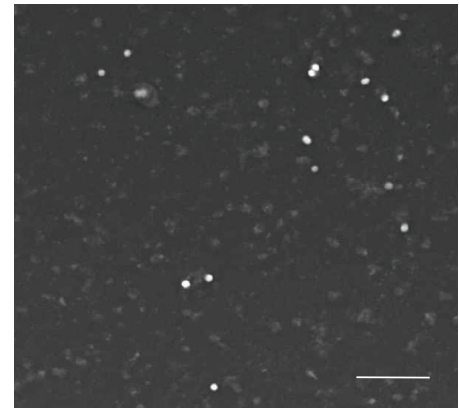

(f)

FIGURE 3: Scanning electron microscopy of MRSA biofilms grown in ethanol extract of R. javanica. (a) Control; (b) $0.01 \mathrm{mg} / \mathrm{mL}$; (c) $0.05 \mathrm{mg} / \mathrm{mL}$; (d) $0.1 \mathrm{mg} / \mathrm{mL}$; (e) $0.2 \mathrm{mg} / \mathrm{mL}$; (f) positive control (0.1\% NaF); bar = $10 \mu \mathrm{m}$. 

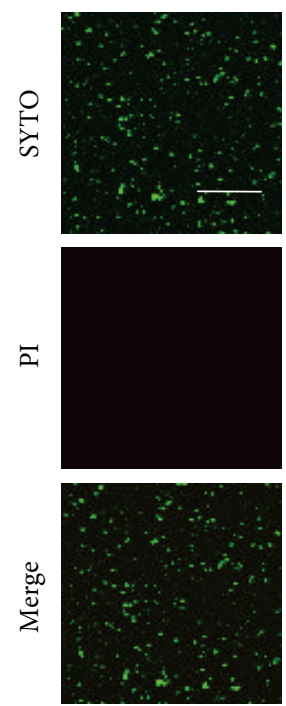

(a)
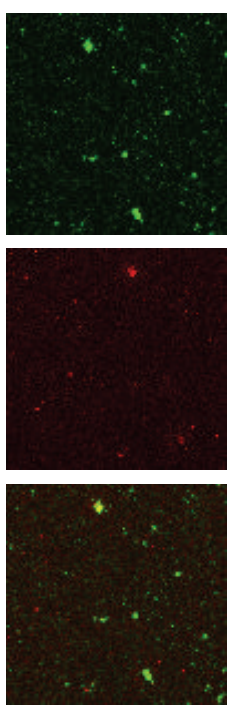

(b)
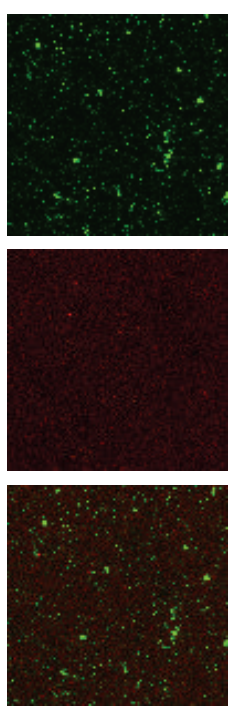

(c)
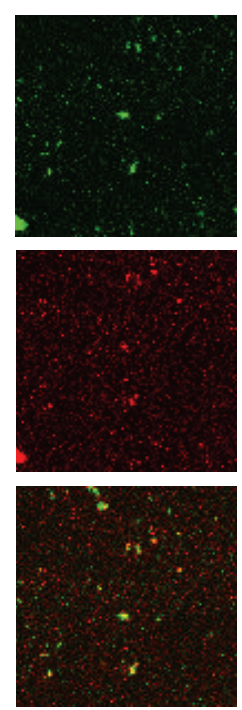

(d)
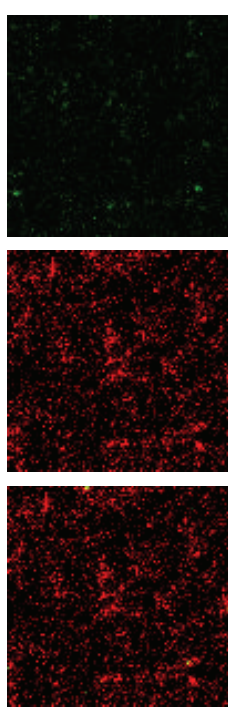

(e)
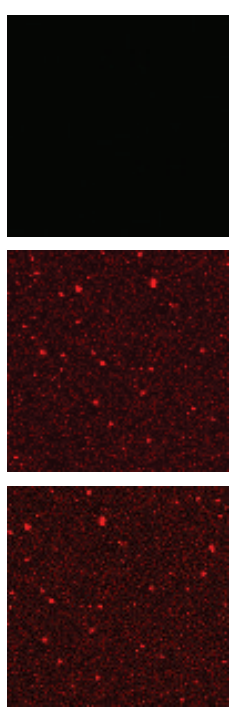

(f)

Figure 4: Bactericidal effect of ethanol extract of $R$. javanica. Cultured MRSA was treated with high concentration $(0.2-1.6 \mathrm{mg} / \mathrm{mL})$ of $R$. javanica extract and stained with LIVE/DEAD BacLight Bacterial Viability Kit. The stained bacteria were observed by confocal laser scanning microscopy. Treatment with ethanol extract of $R$. javanica decreased green-labeled living bacteria (SYTO 9 stain) and increased red-labeled dead bacteria (PI stain) in a dose-dependent manner. (a) Control; (b) $0.2 \mathrm{mg} / \mathrm{mL}$; (c) $0.4 \mathrm{mg} / \mathrm{mL}$; (d) $0.8 \mathrm{mg} / \mathrm{mL}$; (e) $1.6 \mathrm{mg} / \mathrm{mL}$; (f) positive control $(0.1 \% \mathrm{NaF})$; bar $=50 \mu \mathrm{m}$.

3.5. Inhibitory Effect of R. javanica on Virulence Factor Gene Expression. Real-time PCR analysis was performed to examine the effect of $R$. javanica extract on the genetic expression of virulence factors in MRSA (Figure 5). The expression of $m e c A$, sea, agrA, and sarA was significantly decreased in MRSA $(P<0.05)$ when it was treated with $R$. javanica extract.

3.6. Phytochemical Analysis. The results of the phytochemical tests conducted on the ethanol extract of $R$. javanica are shown in Table 3. Preliminary phytochemical analyses revealed the strong presence of phenolics, the moderate presence of glycosides, and the weak presence of flavonoids, steroids (terpenoids), and organic acids. These results suggest that phenolics may have been responsible for the antibacterial activity observed in the present study.

\section{Discussion}

In the present study, we examined the antimicrobial activity of $R$. javanica extract against MRSA. Our data show that 0.05$0.2 \mathrm{mg} / \mathrm{mL}$ of the ethanol extract of $R$. javanica inhibited the growth of MRSA. The fact that the extract of $R$. javanica inhibited MRSA growth provides a scientific rationale for the use of this extract by local inhabitants as an antimicrobial agent. Traditionally, $R$. javanica has been used to treat dysentery and diarrhea in Korea, China, and Japan [15].

Previous studies have shown that MRSA is able to metabolize dietary carbohydrates and thereby produce organic acids $[10,11]$. The major organic acid produced by MRSA is acetic acid, which can lower the $\mathrm{pH}$ of infected regions and contribute to the formation of firmly adhering biofilm-like microbial communities [10]. In the present study, the extract of $R$. javanica inhibited the decrease of $\mathrm{pH}$ induced by MRSA.
This result suggests that the extract of $R$. javanica may inhibit carbohydrate metabolism in MRSA.

MRSA has the ability to adhere, colonize, and form biofilms on damaged tissue, implanted medical devices, and prosthetic devices $[7,8]$. Biofilms are surface-associated bacterial communities on biological or abiotic substrata. They are enclosed firmly in a self-produced extracellular matrix composed of polysaccharides and proteins. Biofilms are very difficult to remove and are a source of refractory infections. Biofilm formation on the surface of implanted medical devices frequently requires surgical removal of the biofilm, debridement of the surrounding tissue, and long-term antibiotic treatment $[7,8]$. The most well-known assay method for the detection of biofilm formation is the tissue culture plate assay method [9]. In our study, the extract of $R$. javanica inhibited biofilm formation by MRSA at concentrations ranging from 0.05 to $0.1 \mathrm{mg} / \mathrm{mL}$, as seen by safranin staining. SEM data of MRSA biofilm formation were consistent with the data from safranin staining. However, the data on biofilm culture were different from the results of planktonic cell growth measurements. The growth of MRSA in planktonic culture was inhibited at concentrations higher than $0.05 \mathrm{mg} / \mathrm{mL}$. A previous study has shown that bacteria in biofilm cultures are physiologically different from planktonic cells of the same organism. Biofilm formation enhances bacterial resistance to both the host defense system and antimicrobials [26]. However, in our study, bacterial resistance against $R$. javanica may be similar within both biofilm cultures and planktonic cultures.

Bactericidal effect of $R$. javanica extract was tested by confocal laser scanning microscopy. Bacterial viability was gradually decreased at high concentration $(0.4-1.6 \mathrm{mg} / \mathrm{mL})$ of $R$. javanica extract in a dose-dependent manner. This result 


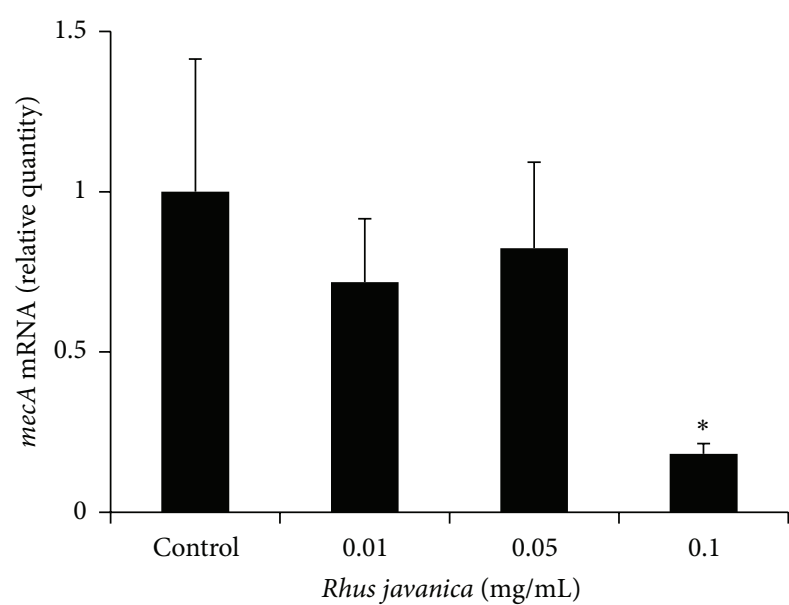

(a)

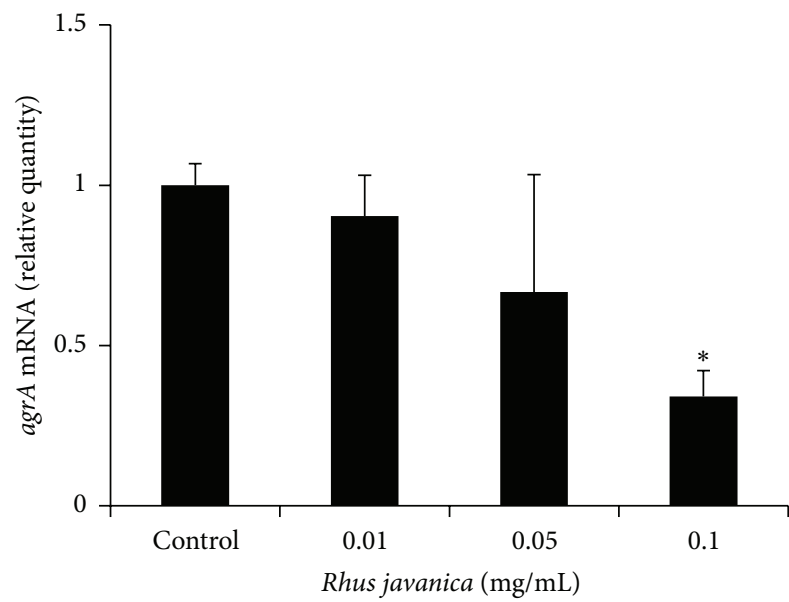

(c)

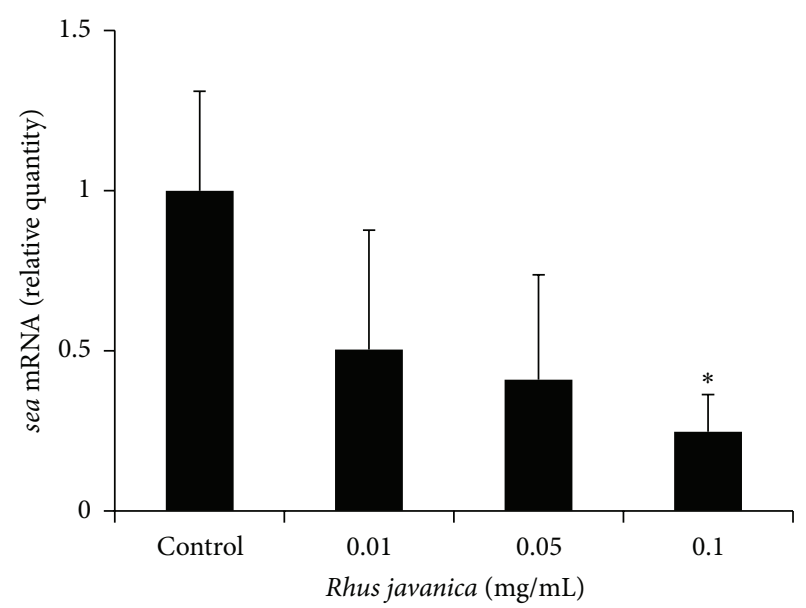

(b)

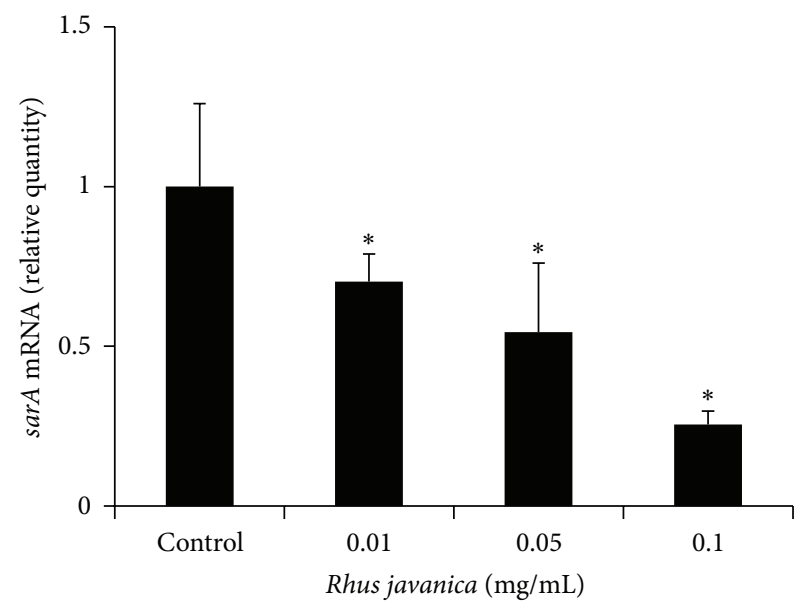

(d)

FIGURE 5: Real-time PCR analysis of expression of several virulence factor genes. MRSA was cultured and treated with sub minimal inhibitory concentration $(0.01-0.1 \mathrm{mg} / \mathrm{mL})$ of $R$. javanica extract, and real-time PCR analysis was then performed as described in Section 2 . mecA, sea, and agrA expression was significantly inhibited at $0.1 \mathrm{mg} / \mathrm{mL}$ of $R$. javanica extract, and sarA was significantly inhibited at concentration higher than $0.01 \mathrm{mg} / \mathrm{mL}$. Each value is expressed as a mean \pm standard deviation. Significance was determined at ${ }^{*} P<0.05$ when compared with the control.

TABLE 3: Phytochemical analysis of the ethanol extract of $R$. javanica.

\begin{tabular}{lc}
\hline Plant constituents & Ethanol extract \\
\hline Alkaloids & - \\
Phenolics & +++ \\
Flavonoids & + \\
Glycosides & ++ \\
Peptides & - \\
Steroids, terpenoids & + \\
Organic acids & + \\
\hline
\end{tabular}

+++: strong; ++: moderate; +: weak; -: absent.

suggests that high concentration of $R$. javanica extract may be bactericidal on MRSA.

An antibiotic resistance gene, mecA encodes $\mathrm{PBP} 2 \mathrm{a}$, which has low affinity to $\beta$-lactam antibiotics, so it allow cellwall biosynthesis despite the presence of $\beta$-beta-lactams [2].
In the present study, the effect of $R$. javanica extract on the genetic expression of $m e c A$ was determined by real-time PCR analysis. The expression of mecA was significantly decreased in MRSA when it was treated with $R$. javanica extract.

A virulence factor gene, sea encodes Staphylococcal enterotoxin A which is one of major virulence factors in MRSA [27]. Staphylococcal enterotoxin A is one cause of gastroenteritis in human and acts as a superantigen. In this study, the $R$. javanica extract significantly inhibited sea expression. sea gene expression in MRSA is regulated by global regulators such as agr and sarA gene [21]. In our study, $R$. javanica extract showed the inhibition of agrA and sarA expressionin MRSA. agrA encodes accessory gene regulator A which positively regulates exotoxin-encoding genes. sar A also upregulates expression of virulence factor genes. Previous research has shown that inhibition of agrA or sarA expression by some chemicals such as thymol or clindamycin reduces transcription of exotoxin-encoding genes [21]. 
In the present study, suppressive effect of $R$. javanica extract on sea gene expression may, in part, be related with inhibitory effect of $R$. javanica extract on agrA and sarA expression [28].

Previous reports indicate that the leaves of $R$. javanica contain mainly tannins (50-70\%) such as tannic acid, pyrogallol, gallic methy lester, syringic acid, protocatechuic acid, and $1,2,3,4,6$-penta- $O$-galloyl- $\beta$-D-glucose $[14,29,30]$. In the present study, we found the strong presence of phenolics, the moderate presence of glycosides, and the weak presence of flavonoids, steroids (terpenoids), and organic acids. These results suggest that phenolics may have been responsible for the antibacterial activity observed in the present study. However, further studies are needed to elucidate the antimicrobial principles underlying the action against MRSA.

In conclusion, our study demonstrated the antimicrobial activity of the ethanol extract of $R$. javanica against MRSA. $R$. javanica inhibited the growth, acid production, and biofilm formation of MRSA. R. javanica also showed bactericidal effect and the inhibition of the genetic expression of virulence factors such as mecA, sea, agrA, and sarA. Phytochemical analysis revealed the strong presence of phenolics. These results suggest that $R$. javanica may be a useful medicinal plant for inhibiting MRSA, which may be related to the presence of phenolics in the R. javanica extract.

\section{Conflict of Interests}

The authors declare that they have no conflict of interests.

\section{Authors' Contribution}

Y.-O. You and N.-Y. Choi contributed equally to this work.

\section{Acknowledgment}

This research was supported by Basic Science Research Program through the National Research Foundation of Korea (NRF) funded by the Ministry of Education, Science and Technology (no. 2012R1A1A4A01012680).

\section{References}

[1] A. Al-Habib, E. Al-Saleh, A.-M. Safer, and M. Afzal, "Bactericidal effect of grape seed extract on methicillin-resistant Staphylococcus aureus (MRSA)," Journal of Toxicological Sciences, vol. 35, no. 3, pp. 357-364, 2010.

[2] C. C. S. Fuda, J. F. Fisher, and S. Mobashery, " $\beta$-Lactam resistance in Staphylococcus aureus: the adaptive resistance of a plastic genome," Cellular and Molecular Life Sciences, vol. 62, no. 22, pp. 2617-2633, 2005.

[3] L. B. Rice, "Antimicrobial resistance in gram-positive bacteria," American Journal of Medicine, vol. 119, no. 6, pp. S11-S19, 2006.

[4] H. Tsuchiya, M. Sato, T. Miyazaki et al., "Comparative study on the antibacterial activity of phytochemical flavanones against methicillin-resistant Staphylococcus aureus," Journal of Ethnopharmacology, vol. 50, no. 1, pp. 27-34, 1996.

[5] S. Stefani and P. E. Varaldo, "Epidemiology of methicillinresistant staphylococci in Europe," Clinical Microbiology and Infection, vol. 9, no. 12, pp. 1179-1186, 2003.
[6] H. B. Kim, H.-C. Jang, H. J. Nam et al., "In vitro Activities of 28 antimicrobial agents against Staphylococcus aureus isolates from tertiary-care hospitals in Korea: A Nationwide Survey," Antimicrobial Agents and Chemotherapy, vol. 48, no. 4, pp. 11241127, 2004.

[7] C. L. Quave, L. R. W. Plano, T. Pantuso, and B. C. Bennett, "Effects of extracts from Italian medicinal plants on planktonic growth, biofilm formation and adherence of methicillinresistant Staphylococcus aureus," Journal of Ethnopharmacology, vol. 118, no. 3, pp. 418-428, 2008.

[8] D. G. Conrady, C. C. Brescia, K. Horii, A. A. Weiss, D. J. Hassett, and A. B. Herr, "A zinc-dependent adhesion module is responsible for intercellular adhesion in staphylococcal biofilms," Proceedings of the National Academy of Sciences of the United States of America, vol. 105, no. 49, pp. 19456-19461, 2008.

[9] P. Jia, Y. J. Xue, X. J. Duan, and S.-H. Shao, "Effect of cinnamaldehyde on biofilm formation and sarA expression by methicillinresistant Staphylococcus aureus," Letters in Applied Microbiology, vol. 53, no. 4, pp. 409-416, 2011.

[10] A. G. O. Manetti, T. Köller, M. Becherelli et al., "Environmental acidification drives $\mathrm{S}$. pyogenes pilus expression and microcolony formation on epithelial cells in a FCT-dependent manner," PLoS ONE, vol. 5, no. 11, Article ID el3864, 2010.

[11] T. M. Rode, T. Møretrø, S. Langsrud, Ø. Langsrud, G. Vogt, and A. Holck, "Responses of Staphylococcus aureus exposed to HCL and organic acid stress," Canadian Journal of Microbiology, vol. 56, no. 9, pp. 777-792, 2010.

[12] S. Faizi, N. R. Mughal, R. A. Khan et al., "Evaluation of the antimicrobial property of polyalthia iongifolia var. Pendula: isolation of a lactone as the active antibacterial agent from the ethanol extract of the stem," Phytotherapy Research, vol. 17, no. 10, pp. 1177-1181, 2003.

[13] S. Gibbons, J. Leimkugel, M. Oluwatuyi, and M. Heinrich, "Activity of Zanthoxylum clava-herculis extracts against multidrug resistant methicillin-resistant Staphylococcus aureus (mdrMRSA)," Phytotherapy Research, vol. 17, no. 3, pp. 274-275, 2003.

[14] B. C. Cha, S. B. Lee, T. J. Rhim, and K. H. Lee, "Constituents of antioxidative activity and free radical scavenging effect from Galla Rhois (Rhus javanica Linne)," Korean Journal of Pharmacognosy, vol. 31, no. 2, pp. 185-189, 2000.

[15] I. S. L. Im Seon Lee, S.-R. Oh, K.-S. Ahn, and H.-K. Lee, "Semialactone, isofouquierone peroxide and fouquierone, three new dammarane triterpenes from Rhus javanica," Chemical and Pharmaceutical Bulletin, vol. 49, no. 8, pp. 1024-1026, 2001.

[16] K.-J. Kim, Y.-H. Kim, H.-H. Yu et al., "Antibacterial activity and chemical composition of essential oil of Chrysanthemum boreale," Planta Medica, vol. 69, no. 3, pp. 274-277, 2003.

[17] M. Matsumoto, T. Minami, H. Sasaki, S. Sobue, S. Hamada, and T. Ooshima, "Inhibitory effects of oolong tea extract on cariesinducing properties of mutans streptococci," Caries Research, vol. 33, no. 6, pp. 441-445, 1999.

[18] E. O’Neill, H. Humphreys, and J. P. O'Gara, "Carriage of both the fnbA and fnbB genes and growth at $37 \circ \mathrm{C}$ promote FnBPmediated biofilm development in meticillin-resistant Staphylococcus aureus clinical isolates," Journal of Medical Microbiology, vol. 58, no. 4, pp. 399-402, 2009.

[19] F. C. Petersen, D. Pecharki, and A. A. Scheie, "Biofilm mode of growth of Streptococcus intermedius favored by a competencestimulating signaling peptide," Journal of Bacteriology, vol. 186, no. 18, pp. 6327-6331, 2004.

[20] K. Nakamiya, S. Hashimoto, H. Ito, J. S. Edmonds, A. Yasuhara, and M. Morita, "Microbial treatment of bis (2-ethylhexyl) 
phthalate in polyvinyl chloride with isolated bacteria," Journal of Bioscience and Bioengineering, vol. 99, no. 2, pp. 115-119, 2005.

[21] J. Qiu, X. Zhang, M. Luo et al., "Subinhibitory concentrations of perilla oil affect the expression of secreted virulence factor genes in Staphylococcus aureus," PLoS ONE, vol. 6, no. 1, Article ID e16160, 2011.

[22] P. Jia, Y. J. Xue, X. J. Duan, and S.-H. Shao, "Effect of cinnamaldehyde on biofilm formation and sarA expression by methicillinresistant Staphylococcus aureus," Letters in Applied Microbiology, vol. 53, no. 4, pp. 409-416, 2011.

[23] A. E. Rosato, W. A. Craig, and G. L. Archer, "Quantitation of mecA transcription in oxacillin-resistant Staphylococcus aureus clinical isolates," Journal of Bacteriology, vol. 185, no. 11, pp. 3446-3452, 2003.

[24] P. J. Houghton and A. Raman, Laboratory Handbook for the Fractionation of Natural Extracts, Chapman \& Hall, London, UK, 1998.

[25] W. S. Woo, Experimental Methods for Phytochemistry, Seoul National University Press, Seoul, Korea, 2001.

[26] A. Yoshida and H. K. Kuramitsu, "Multiple Streptococcus mutans genes are involved in biofilm formation," Applied and Environmental Microbiology, vol. 68, no. 12, pp. 6283-6291, 2002.

[27] J. Qiu, D. Wang, H. Xiang et al., "Subinhibitory concentrations of thymol reduce enterotoxins A and B and alpha-hemolysin production in Staphylococcus aureus isolates," PloS One, vol. 5, no. 3, article e9736, 2010.

[28] L. I. Kupferwasser, M. R. Yeaman, C. C. Nast et al., "Salicylic acid attenuates virulence in endovascular infections by targeting global regulatory pathways in Staphylococcus aureus," Journal of Clinical Investigation, vol. 112, no. 2, pp. 222-233, 2003.

[29] Pharmacognosy Research Association, Modern Pharmacognosy, Hakchangsa, Seoul, Korea, 1998.

[30] C. S. Chun, Effects of Rhus javanica extracts on the radical scavenging activities and the oxidative DNA damage in HepG2 cells using the comet assay [M.S. thesis], Andong National University, 2001. 


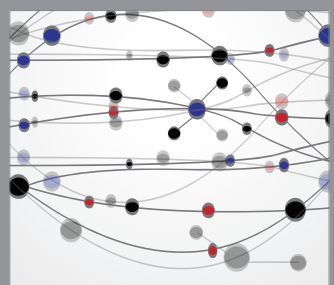

The Scientific World Journal
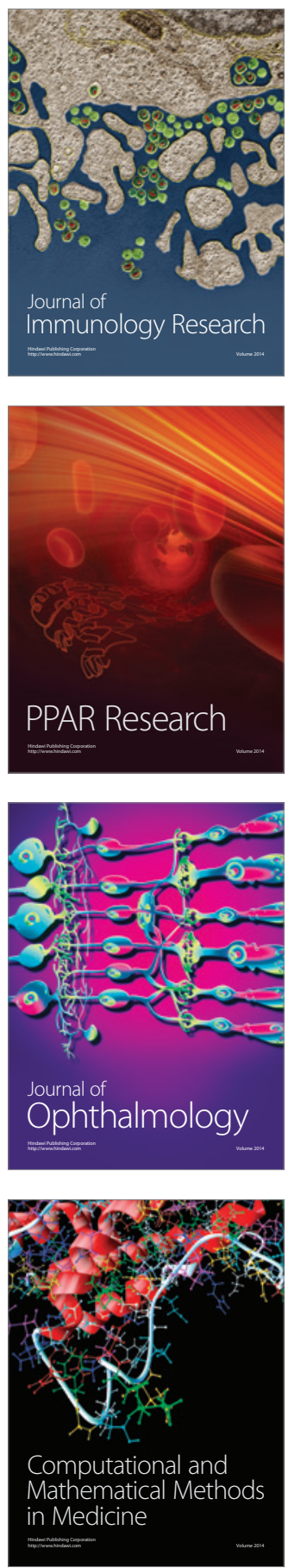

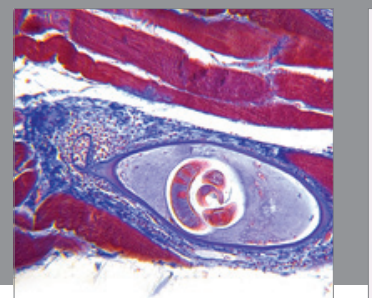

Gastroenterology

Research and Practice
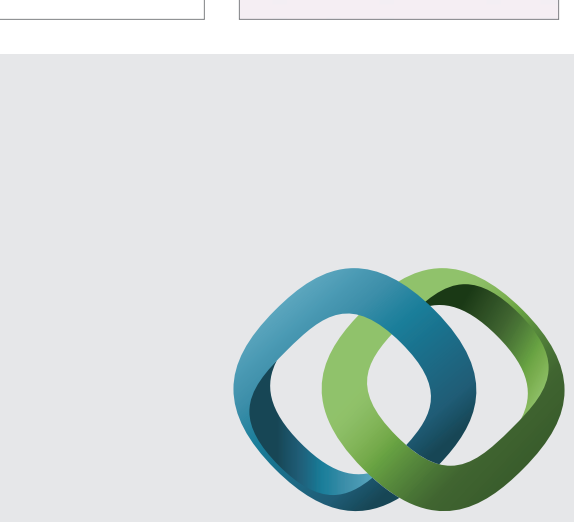

\section{Hindawi}

Submit your manuscripts at

http://www.hindawi.com
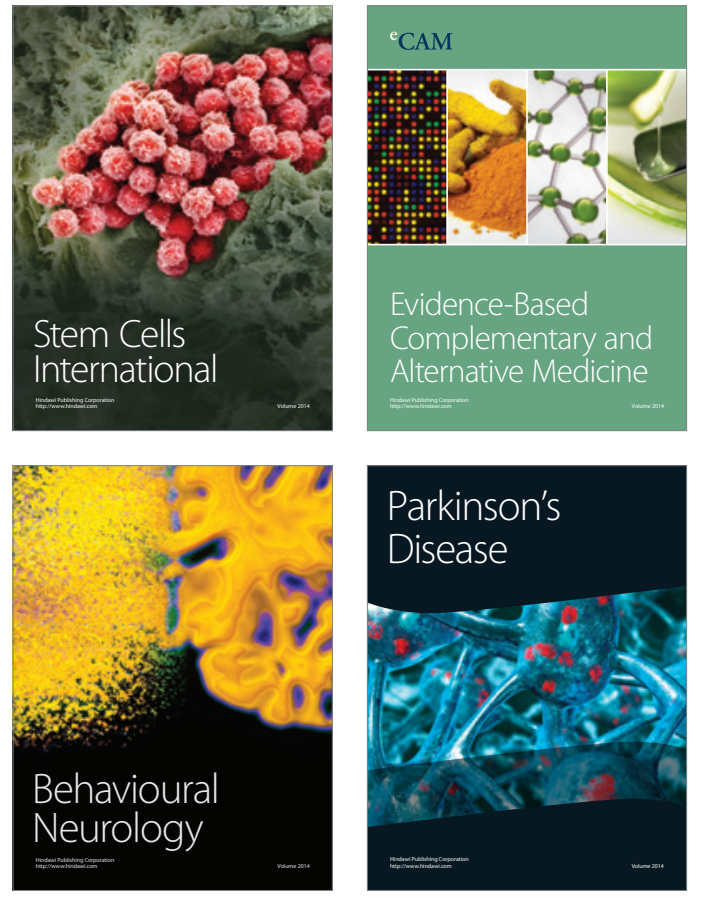
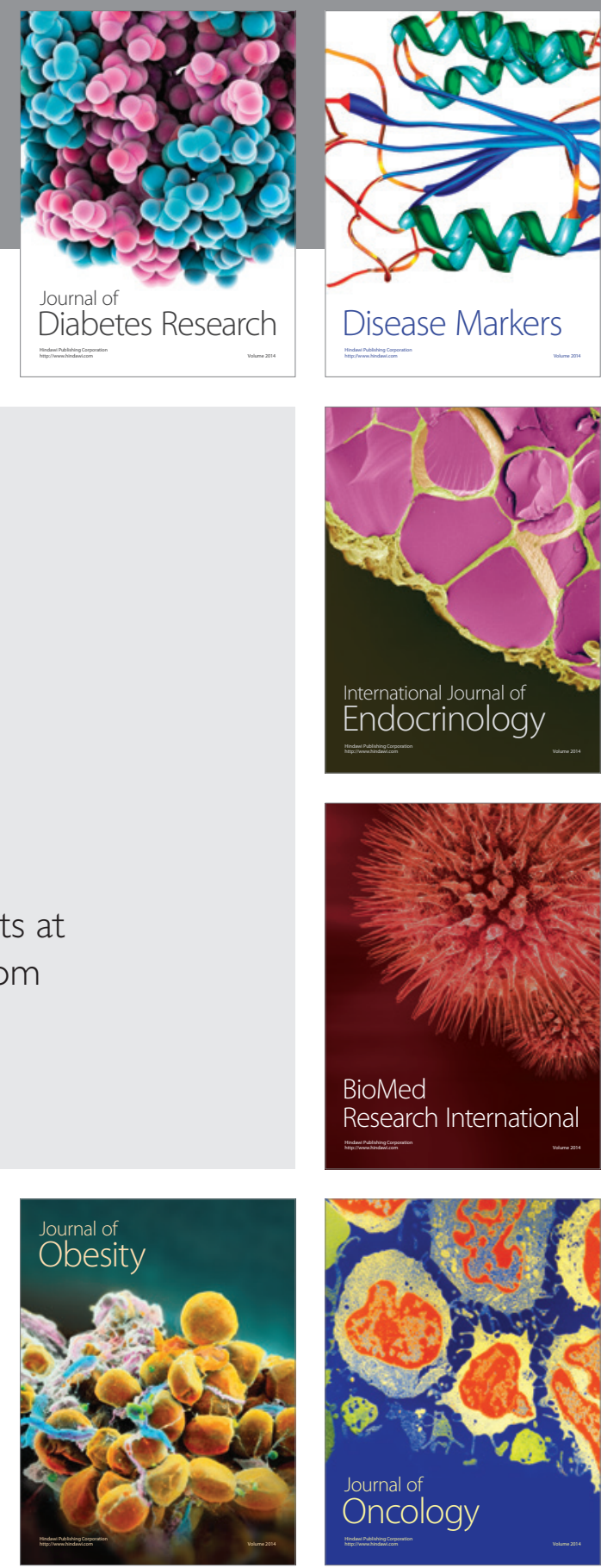

Disease Markers
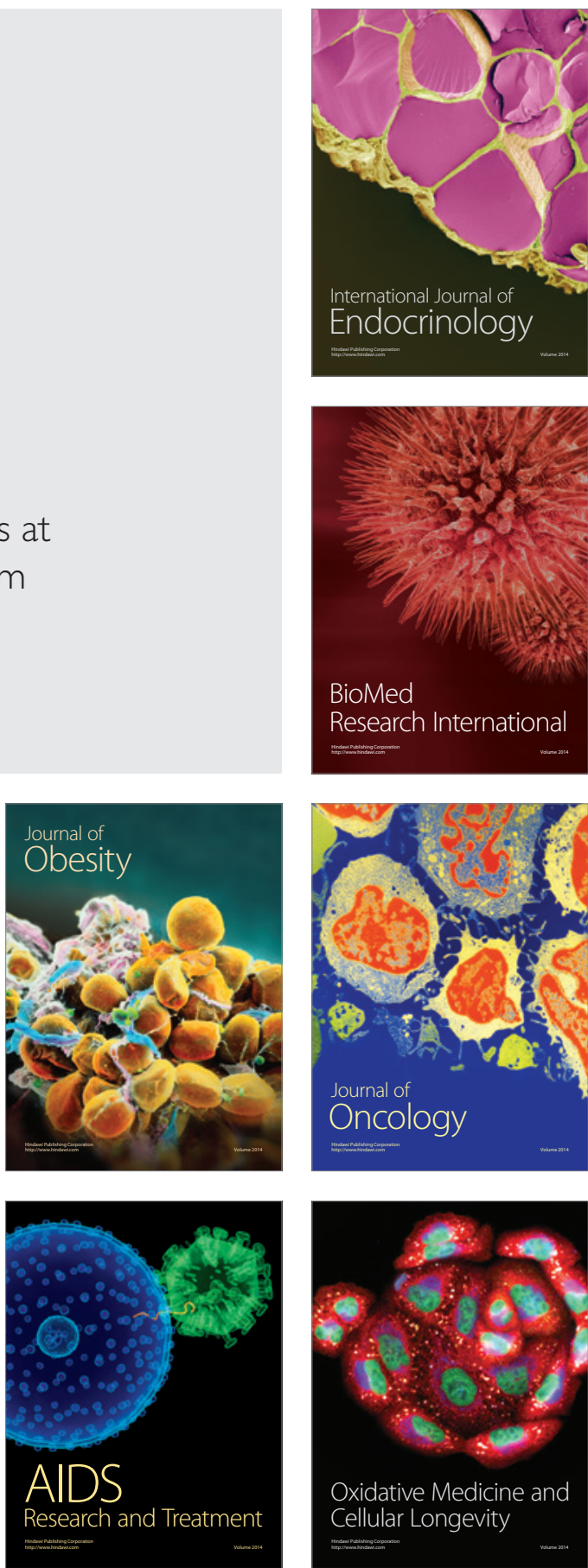